\title{
Two photon decay rate of the Higgs boson in the Inert Doublet Model
}

\section{Bogumiła Świeżewska*}

Faculty of Physics, University of Warsaw

E-mail: Bogumila.Swiezewska@fuw.edu.pl

\section{Maria Krawczyk}

Faculty of Physics, University of Warsaw

E-mail: Maria.Krawczykefuw.edu.pl

\begin{abstract}
Motivated by experimental hints on possible deviations from the SM predictions for the SMlike Higgs boson loop-induced decays, a thorough analysis of the decay rate of the Higgs boson to $\gamma \gamma$ in the Inert Doublet Model (IDM) is performed. Wide set of constraints is taken into account, including also the condition for the existence of the Inert vacuum, which significantly reduces the viable parameter space. The consequences of deviations from SM predictions for the two-photon decay channel for the masses of the charged and neutral scalars (in particular the Dark Matter (DM) candidate), and their couplings to the Higgs boson are analyzed. We find that an enhancement of the two-photon decay rate at the level of 1.2 or greater implies that the charged scalar and the DM candidate are fairly light, with the following bounds on their masses: $62.5 \mathrm{GeV}<M_{D M}<154 \mathrm{GeV}, 70 \mathrm{GeV}<M_{H^{ \pm}}<154 \mathrm{GeV}$.
\end{abstract}

International Conference on the Structure and the Interactions of the Photon including the 20th International Workshop on Photon-Photon Collisions and the International Workshop on High Energy Photon Linear Colliders

20 - 24 May 2013

Paris, France

* Speaker. 


\section{Introduction}

The $h \rightarrow \gamma \gamma$ decay is one of the most important observational channels of the Higgs boson at the LHC. It gained much interest when some deviations from the Standard Model (SM) prediction for its rate have been reported, the current experimental results for the $h \rightarrow \gamma \gamma$ decay rate (signal strength) read: $R_{\gamma \gamma}=1.55_{-0.28}^{+0.33}$ (ATLAS) [1], $R_{\gamma \gamma}=0.77 \pm 0.27$ (CMS) [2].

The $\gamma \gamma$ signal strength is interesting, because it is sensitive to new physics - in particular existence of new charged particles. By this means it is well suited to study Two Higgs Doublet Models (2HDM), distinctive feature of which is the existence of a charged scalar. Precise measurement of the decay rate of the $h \rightarrow \gamma \gamma$ channel can also constrain the invisible decays branching ratios, which would bring information about the masses of extra scalars present in some versions of 2HDMs [3-5].

We analyze the $h \rightarrow \gamma \gamma$ rate in the Inert Doublet Model (IDM), which is a $\mathbb{Z}_{2}$-symmetric version of a $2 \mathrm{HDM}$ with one "inert" doublet, i.e., having zero vacuum expectation value (VEV) and no coupling to fermions at the tree level. It is a very simple extension of the SM, having very rich phenomenology with a good Dark Matter (DM) candidate.

\section{Model}

The IDM is a $2 \mathrm{HDM}$, with the scalar doublets $\Phi_{S}$ and $\Phi_{D}$ [6-8]. It is described by the following Lagrangian:

$$
\mathscr{L}=\mathscr{L}_{\mathrm{gf}}^{\mathrm{SM}}+\mathscr{L}_{\mathrm{Y}}+\mathscr{L}_{\mathrm{H}},
$$

where $\mathscr{L}_{\mathrm{gf}}^{\mathrm{SM}}$ is the SM Lagrangian describing interactions of fermions and gauge bosons, $\mathscr{L}_{\mathrm{Y}}$ is the Yukawa Lagrangian describing interactions of scalars with fermions, and $\mathscr{L}_{\mathrm{H}}$ - Lagrangian of the scalar sector,

$$
\mathscr{L}_{\mathrm{H}}=\left(D^{\mu} \Phi_{S}\right)^{\dagger}\left(D_{\mu} \Phi_{S}\right)+\left(D^{\mu} \Phi_{D}\right)^{\dagger}\left(D_{\mu} \Phi_{D}\right)-V .
$$

The scalar potential $V$ is defined as follows

$$
\begin{aligned}
V= & -\frac{1}{2}\left[m_{11}^{2}\left(\Phi_{S}^{\dagger} \Phi_{S}\right)+m_{22}^{2}\left(\Phi_{D}^{\dagger} \Phi_{D}\right)\right]+\frac{1}{2}\left[\lambda_{1}\left(\Phi_{S}^{\dagger} \Phi_{S}\right)^{2}+\lambda_{2}\left(\Phi_{D}^{\dagger} \Phi_{D}\right)^{2}\right] \\
& +\lambda_{3}\left(\Phi_{S}^{\dagger} \Phi_{S}\right)\left(\Phi_{D}^{\dagger} \Phi_{D}\right)+\lambda_{4}\left(\Phi_{S}^{\dagger} \Phi_{D}\right)\left(\Phi_{D}^{\dagger} \Phi_{S}\right)+\frac{1}{2} \lambda_{5}\left[\left(\Phi_{S}^{\dagger} \Phi_{D}\right)^{2}+\left(\Phi_{D}^{\dagger} \Phi_{S}\right)^{2}\right],
\end{aligned}
$$

with all parameters real and $\lambda_{5}<0$. In the IDM only $\Phi_{S}$ couples to fermions (type I of Yukawa interactions), so $\mathscr{L}_{\mathrm{Y}}$ looks like in the $\mathrm{SM}$ with $\Phi \rightarrow \Phi_{S}$.

During electroweak symmetry breaking only $\Phi_{S}$ acquires non-zero VEV: $\left\langle\Phi_{S}\right\rangle=\left(\begin{array}{c}0 \\ v / \sqrt{2}\end{array}\right)$, $\left\langle\Phi_{D}\right\rangle=0, v=246 \mathrm{GeV}$. The particle spectrum consists of $h-$ a SM-like Higgs boson originating from $\Phi_{S}$ and four inert (dark) scalars: $H, A$ and $H^{ \pm}$, which originate from $\Phi_{D}$ and thus do not have tree-level couplings to fermions.

Note that the whole Lagrangian as well as the vacuum state are invariant under the $D$ symmetry:

$$
\Phi_{D} \stackrel{D}{\rightarrow}-\Phi_{D}, \quad \Phi_{S} \stackrel{D}{\rightarrow} \Phi_{S}, \quad \Phi_{\mathrm{SM}} \stackrel{D}{\rightarrow} \Phi_{\mathrm{SM}} .
$$

Hence, the lightest $D$-odd particle $(H)$ is stable, providing a DM candidate. It has been shown that the DM in the IDM can give the relic density in agreement with the WMAP measurements 
$\left(0.1018<\Omega_{D M} h^{2}<0.1234\right.$ at $3 \sigma$ confidence level) when its mass is in one of the following three regions [10-12]: low $\left(M_{H} \lesssim 10 \mathrm{GeV}\right)$, intermediate $\left(40 \mathrm{GeV} \lesssim M_{H} \lesssim 150 \mathrm{GeV}\right)$ or heavy $\left(M_{H} \gtrsim\right.$ $500 \mathrm{GeV})$.

\section{Constraints}

In this analysis we take into account the following constraints

Vacuum stability For a stable vacuum state to exist it is necessary that the scalar potential $V$ is bounded from below. This leads to the following constraints: $\lambda_{1}>0, \lambda_{2}>0, \lambda_{3}+$ $\sqrt{\lambda_{1} \lambda_{2}}>0, \quad \lambda_{345}+\sqrt{\lambda_{1} \lambda_{2}}>0$, where $\lambda_{345}=\lambda_{3}+\lambda_{4}+\lambda_{5}[6]$.

These are the tree-level conditions for the stability of the potential. It is known, that in the SM the loop corrections from the top quark can render the effective potential negative, leading to an instability. The analysis of the effective potential for the IDM is beyond the scope of this paper. However, it has been shown [5] that the positive loop contributions of the scalar particles can partially cancel the top contribution, allowing the IDM to be valid up to high energy scales (even at the level of the Planck energy).

Perturbative unitarity Eigenvalues $\Lambda_{i}$ of the high-energy scattering matrix (for the $2 \rightarrow 2$ processes) are required to fulfill the condition $\left|\Lambda_{i}\right|<8 \pi$. This leads to upper bounds on the parameters of the potential and the masses of the scalars, in particular $\lambda_{2} \leqslant 8.38$.

Existence of the Inert vacuum Inert state is a global minimum of the scalar potential if the matrix of the second derivatives of $V$ is positive definite (condition for local minimum), and $\frac{m_{11}^{2}}{\sqrt{\lambda_{1}}}>$ $\frac{m_{22}^{2}}{\sqrt{\lambda_{2}}}$ (energy of the Inert vacuum is lower than energy of a coexisting minimum). The last condition, when combined with unitarity bound on $\lambda_{2} \leqslant 8.38$ and the value of the Higgs boson mass $M_{h}^{2}=m_{11}^{2}=\lambda_{1} v^{2}=(125 \mathrm{GeV})^{2}$, gives an upper bound on the mass parameter $m_{22}^{2}[13]:$

$$
m_{22}^{2} \lesssim 9 \cdot 10^{4} \mathrm{GeV}^{2}
$$

$H$ as DM candidate As $H$ is the DM candidate it has to be the lightest of the dark scalars: $M_{H}<$ $M_{A}, M_{H^{ \pm}}$.

Electroweak Precision Tests (EWPT) The values of $S$ and $T$ parameters are required to lie within $2 \sigma$ ellipses in the $(S, T)$ plane with the central values: $S=0.03 \pm 0.09, T=0.07 \pm 0.08$ and correlation equal to $87 \%$ [14].

LEP bounds The scalars' masses are constrained by the LEP measurements as follows

$$
M_{H^{ \pm}}+M_{H}>M_{W}, \quad M_{H^{ \pm}}+M_{A}>M_{W}, \quad M_{H}+M_{A}>M_{Z}, \quad M_{H^{ \pm}}>70 \mathrm{GeV}
$$

while the region where simultaneously $M_{H}<80 \mathrm{GeV}, M_{A}<100 \mathrm{GeV}$ and $M_{A}-M_{H}>8 \mathrm{GeV}$ is excluded $[15,16]$.

LHC We set the mass of the SM-like Higgs boson $h$ to $125 \mathrm{GeV}$. 


\section{Two photon decay rate}

The two-photon decay rate of the Higgs boson is defined as follows [3, 4]:

$$
R_{\gamma \gamma}:=\frac{\sigma(p p \rightarrow h \rightarrow \gamma \gamma)^{\mathrm{IDM}}}{\sigma(p p \rightarrow h \rightarrow \gamma \gamma)^{\mathrm{SM}}} \approx \frac{[\sigma(g g \rightarrow h) \operatorname{Br}(h \rightarrow \gamma \gamma)]^{\mathrm{IDM}}}{[\sigma(g g \rightarrow h) \mathrm{Br}(h \rightarrow \gamma \gamma)]^{\mathrm{SM}}}=\frac{\operatorname{Br}(h \rightarrow \gamma \gamma)^{\mathrm{IDM}}}{\mathrm{Br}(h \rightarrow \gamma \gamma)^{\mathrm{SM}}},
$$

where the small width approximation has been used. Moreover, the facts that the gluon fusion is the main production channel of the Higgs boson and that $\sigma(g g \rightarrow h)^{\mathrm{IDM}}=\sigma(g g \rightarrow h)^{\mathrm{SM}}$ have been taken into account.

As can be seen, both the partial decay width of the Higgs boson to two photons, $\Gamma(h \rightarrow \gamma \gamma)^{\mathrm{IDM}}$, and the total decay width of the Higgs, $\Gamma(h)^{\mathrm{IDM}}$, contribute to $R_{\gamma \gamma}$. There are two possible origins of deviation from $R_{\gamma \gamma}=1$ : the charged scalar loop contributing to $\Gamma(h \rightarrow \gamma \gamma)^{\mathrm{IDM}}$ and the invisible decays augmenting $\Gamma(h)^{\mathrm{IDM}}$.

\subsection{Invisible Higgs decays}

Many channels contribute to the total decay width of the Higgs boson in the IDM, namely

$$
\begin{aligned}
\Gamma(h)= & \Gamma(h \rightarrow b \bar{b})+\Gamma\left(h \rightarrow W W^{*}\right)+\Gamma\left(h \rightarrow \tau^{+} \tau^{-}\right)+\Gamma(h \rightarrow g g)+\Gamma\left(h \rightarrow Z Z^{*}\right)+\Gamma(h \rightarrow c \bar{c}) \\
& +\Gamma(h \rightarrow Z \gamma)+\Gamma(h \rightarrow \gamma \gamma)+\Gamma(h \rightarrow H H)+\Gamma(h \rightarrow A A) .
\end{aligned}
$$

All the partial widths in the first line of Eq. (4.2) are identical to the corresponding SM widths. The $\gamma \gamma$ and $Z \gamma$ partial decay widths are modified with respect to the SM by the presence of the charged scalar loop. However, the $\gamma \gamma$ and $Z \gamma$ branching ratios are of the order of $10^{-2}-10^{-3}$ so their contributions to the total decay width are negligible. Thus the total decay width can deviate from the SM prediction only due to the invisible decay channels.

The decay width of the Higgs boson to two DM particles is given by:

$$
\Gamma(h \rightarrow H H)=\frac{\lambda_{345}^{2} v^{2}}{32 \pi M_{h}} \sqrt{1-\frac{4 M_{H}^{2}}{M_{h}^{2}}} .
$$

For the $h \rightarrow A A$ decay $M_{H}$ is exchanged to $M_{A}$ and $\lambda_{345}$ to $\lambda_{345}^{-}\left(\lambda_{345}^{-}=\lambda_{3}+\lambda_{4}-\lambda_{5}\right)$. The branching ratios of the Higgs boson as functions of $\lambda_{345}$ are shown in Fig. 1 [3]. It can be seen, that the invisible decay channels dominate over the SM decays if they are kinematically allowed. If the invisible channels are closed, the $H^{ \pm}$loop impact on $h \rightarrow \gamma \gamma$ and $h \rightarrow Z \gamma$ becomes clearly visible.

\subsection{Charged scalar loop}

When the invisible channels are closed, important contribution to $R_{\gamma \gamma}$ comes from the charged scalar loop, which can interfere either constructively or destructively with the SM contribution. The partial width of the Higgs decay to two photons is given by $[8,17]$ :

$$
\Gamma(h \rightarrow \gamma \gamma)^{\mathrm{IDM}}=\frac{G_{F} \alpha^{2} M_{h}^{3}}{128 \sqrt{2} \pi^{3}}\left|\mathscr{M}^{\mathrm{SM}}+\frac{2 M_{H^{ \pm}}^{2}+m_{22}^{2}}{2 M_{H^{ \pm}}^{2}} A_{0}\left(\frac{4 M_{H^{ \pm}}^{2}}{M_{h}^{2}}\right)\right|^{2},
$$

the definitions of $\mathscr{M}^{\mathrm{SM}}$ and $A_{0}$ can be found in Refs. [3, 17]. In the above formula $2 M_{H^{ \pm}}^{2}+m_{22}^{2}=$ $\lambda_{3} v^{2}$, where $\lambda_{3}$ is proportional to the $h H^{+} H^{-}$coupling, so one of the following sets of parameters $\lambda_{3}, M_{H^{ \pm}}$or $m_{22}^{2}, M_{H^{ \pm}}$can be chosen to determine $\Gamma(h \rightarrow \gamma \gamma)^{\mathrm{IDM}}$. 

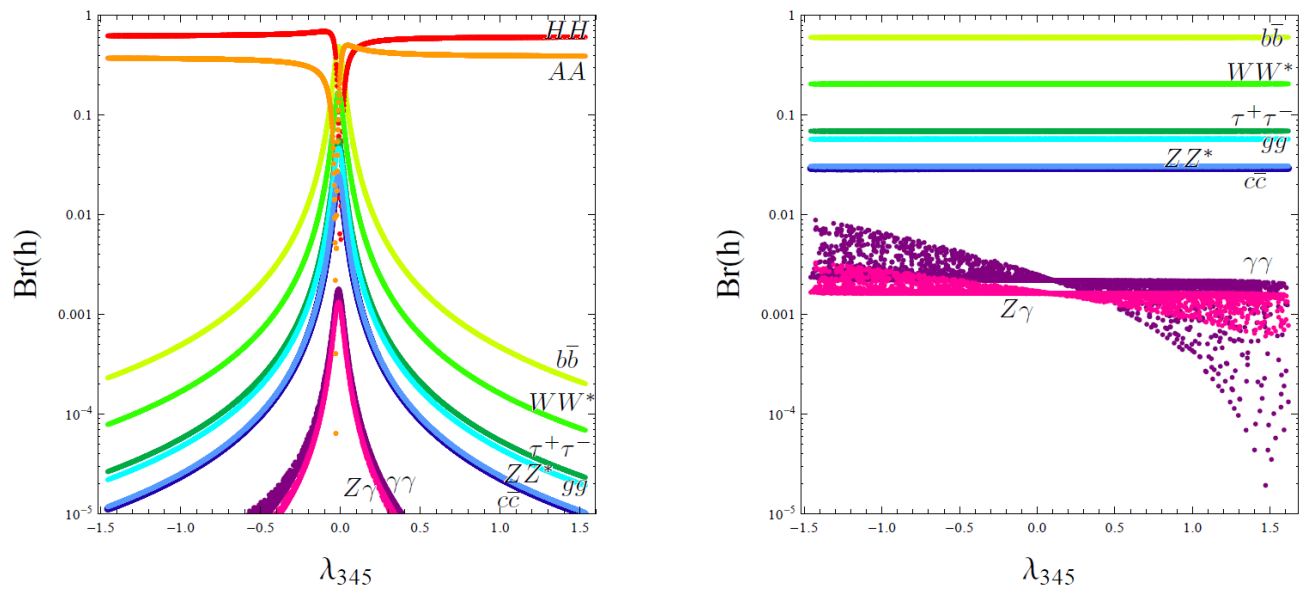

Figure 1: Branching ratios of $h$ as functions of $\lambda_{345}$. Left panel: decay channels $h \rightarrow H H$ and $h \rightarrow A A$ are open $\left(M_{H}=50 \mathrm{GeV}, M_{A}=58 \mathrm{GeV}\right)$, right panel: invisible $h$ decay channels are kinematically closed $\left(M_{H}=75 \mathrm{GeV}, M_{A}>M_{H}\right)$.

If the invisible decay channels are closed, the ratio $R_{\gamma \gamma}$ simplifies to $R_{\gamma \gamma}=\frac{\Gamma(h \rightarrow \gamma \gamma)^{\mathrm{IDM}}}{\Gamma(h \rightarrow \gamma \gamma)^{\mathrm{SM}}}$. Then the inequality $R_{\gamma \gamma}>1$ can be solved analytically, leading to two solutions. Using the lower LEP bound on $M_{H^{ \pm}}: M_{H^{ \pm}}>70 \mathrm{GeV}$ and substituting $M_{h}=125 \mathrm{GeV}$ we get [3].

1. $m_{22}^{2}<-2 M_{H^{ \pm}}^{2}<-9.8 \cdot 10^{3} \mathrm{GeV}^{2}$, this solution corresponds to constructive interference between the SM contribution and the charged scalar loop.

2. $m_{22}^{2}>\frac{M_{h}^{2} \operatorname{Re}\left(\mathscr{M}^{\mathrm{SM}}\right)}{1-\left(\frac{2 M_{H^{ \pm}}}{M_{h}}\right)^{2} \arcsin ^{2}\left(\frac{M_{h}}{2 M_{H^{ \pm}}}\right)}-2 M_{H^{ \pm}}^{2} \gtrsim 1.8 \cdot 10^{5} \mathrm{GeV}^{2}$, corresponding to destructive interference (to have $R_{\gamma \gamma}>1$, the $H^{ \pm}$loops has to be at least twice as big as the SM amplitude). Comparing with Eq. (3.1), it can be seen that this option is excluded by the condition for the stability of the Inert vacuum.

So the only surviving solution for $R_{\gamma \gamma}>1$ corresponds to $m_{22}^{2}<-2 M_{H^{ \pm}}^{2}$, which can be translated to the condition $\lambda_{3}<0$, in agreement with Ref. [4].

\section{Results}

To check in which part of the parameter space the two-photon rate is enhanced, we performed a random scan, varying the parameters in the following ranges:

$$
\begin{aligned}
& M_{h}=125 \mathrm{GeV}, \quad 70 \mathrm{GeV} \leqslant M_{H^{ \pm}} \leqslant 1400 \mathrm{GeV}, \quad 0<M_{A} \leqslant 1400 \mathrm{GeV}, \\
& 0<M_{H}<M_{A}, M_{H^{ \pm}}, \quad-2 \cdot 10^{6} \mathrm{GeV}^{2} \leqslant m_{22}^{2} \leqslant 9 \cdot 10^{4} \mathrm{GeV}^{2}, \quad 0<\lambda_{2} \leqslant 8.4 .
\end{aligned}
$$

and taking into account the constraints described in section 3. In all the plots the light green (gray) regions correspond to $R_{\gamma \gamma}>1$ and the dark ones to $R_{\gamma \gamma}<1$. Note that the presented plots are projections of multidimensional regions onto two-dimensional planes, thus light colored regions can overlap dark colored regions (the light ones are always printed on the top of the dark ones). 


\subsection{Impact of the DM mass on $R_{\gamma \gamma}$}

As described before, the impact of the DM particle on $R_{\gamma \gamma}$ is through the invisible decays. Dependence of $R_{\gamma \gamma}$ on $M_{H}$ is presented in Fig. 2. The plot confirms that $R_{\gamma \gamma}>1$ is only possible if
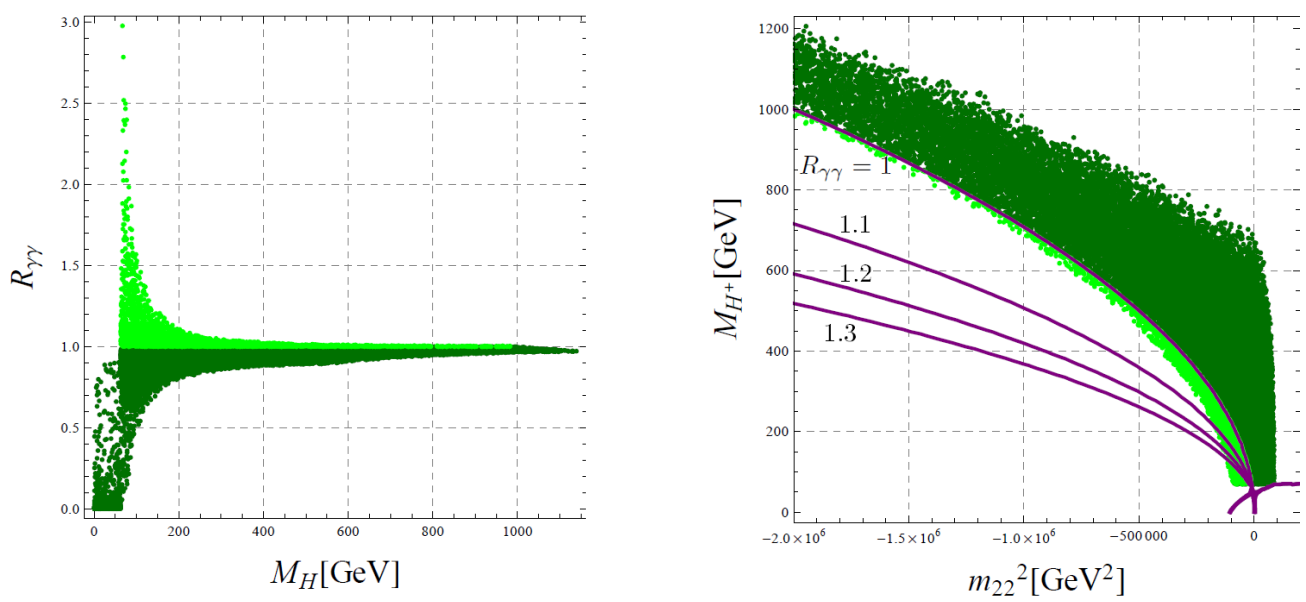

Figure 2: Left panel: $R_{\gamma \gamma}$ versus $M_{H}$. Right panel: region allowed by the constraints described in section 3 in the $\left(m_{22}^{2}, M_{H^{ \pm}}\right)$plane together with the curves indicating constant values of $R_{\gamma \gamma}$ (calculated for invisible channels closed). Light green (gray) corresponds to $R_{\gamma \gamma}>1$, dark green (gray) to $R_{\gamma \gamma}<1$.

$M_{H}>M_{h} / 2 \approx 63 \mathrm{GeV}[3,4]$. As $H$ is the lightest of the dark scalars, then in order to enhance $R_{\gamma \gamma}$ also $M_{A}, M_{H^{ \pm}}>63 \mathrm{GeV}$.

\subsection{Impact of $M_{H^{ \pm}}$on $R_{\gamma \gamma}$}

The possibility of enhancing $R_{\gamma \gamma}$ relies on the parameters $m_{22}^{2}$ and $M_{H^{ \pm}}$. The parameter-space region allowed by the conditions from section 3 in the $\left(m_{22}^{2}, M_{H^{ \pm}}\right)$plane is presented in Fig. 2 (right panel). Also curves indicating constant values of $R_{\gamma \gamma}$ (computed for invisible channels closed, so for $\left.R_{\gamma \gamma}=\frac{\Gamma(h \rightarrow \gamma \gamma)^{\mathrm{IM}}}{\Gamma(h \rightarrow \gamma \gamma)^{\mathrm{SM}}}\right)$ are displayed. First thing to note is that $R_{\gamma \gamma}>1$ for very heavy $H^{ \pm}$with mass up to $1 \mathrm{TeV}$. Even larger masses would appear if the range of $m_{22}^{2}$ was wider. Secondly, it can be seen that assuming $R_{\gamma \gamma}>\delta>1$ for some $\delta$, introduces an upper bound on $M_{H^{ \pm}}$. Moreover, the region where $R_{\gamma \gamma}>\delta>1$ is confined to a constrained range of $m_{22}^{2}$, so this result will not change with altering $m_{22}^{2}$ range. For example $R_{\gamma \gamma}>1.2$ implies $M_{H^{ \pm}} \lesssim 154 \mathrm{GeV}$. As $M_{H}<M_{H^{ \pm}}$and taking into account the lower bounds from $R_{\gamma \gamma}>1$ and LEP we have for $R_{\gamma \gamma}>1.2$ [3]:

$$
63 \mathrm{GeV}<M_{H} \lesssim 154 \mathrm{GeV}, \quad 70 \mathrm{GeV}<M_{H^{ \pm}} \lesssim 154 \mathrm{GeV}
$$

\subsection{Impact of the couplings on $R_{\gamma \gamma}$}

Fig. 3 (left panel) confirms the analytical result saying that $R_{\gamma \gamma}>1$ only for $\lambda_{3}<0$. In the right panel the dependence of $R_{\gamma \gamma}$ on $\lambda_{345} \sim h A A$ is shown, and it can be seen that also $R_{\gamma \gamma}>1$ for $\lambda_{345}<0$. Similarly as in the case of $M_{H^{ \pm}}$, when we demand that $R_{\gamma \gamma}>\delta>1$, stronger bounds on these parameters arise. For example $R_{\gamma \gamma}>1.2$ implies [3]

$$
-1.45<\lambda_{3}, \lambda_{345}<-0.18 \text {. }
$$



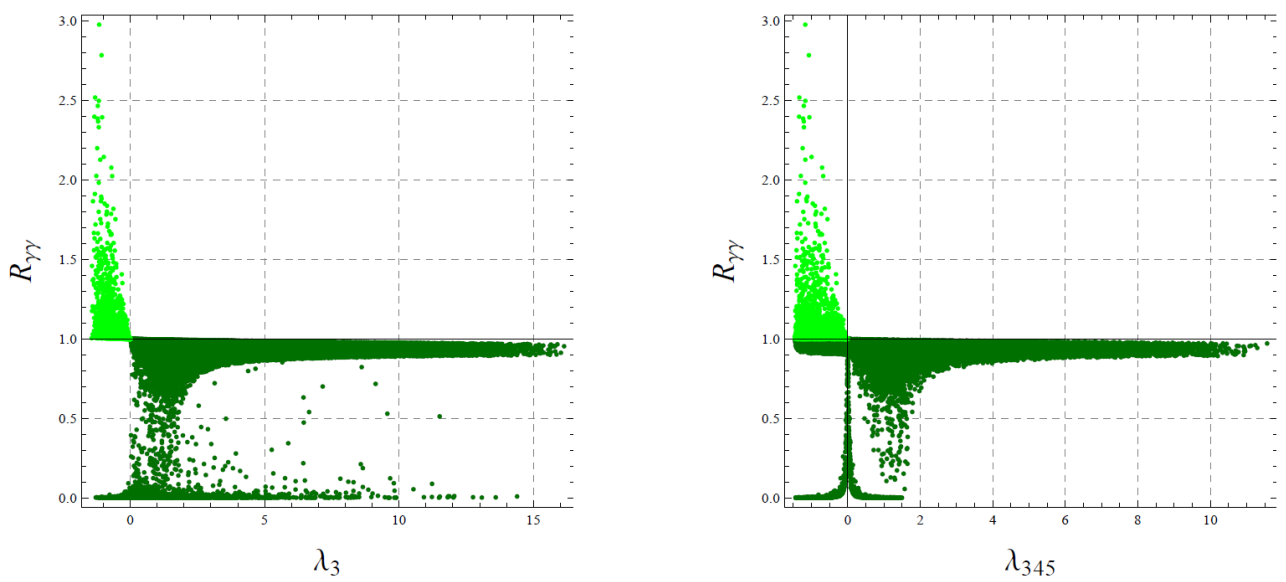

Figure 3: Left panel: $\lambda_{3}$ versus $R_{\gamma \gamma}$. Right panel: $\lambda_{345}$ versus $R_{\gamma \gamma}$. Light green (gray) corresponds to $R_{\gamma \gamma}>1$, dark green (gray) to $R_{\gamma \gamma}<1$.

\section{Summary}

The $h \rightarrow \gamma \gamma$ decay rate, being sensitive to the existence of new charged or neutral scalars, can provide us with new information about the Higgs sector. Within the IDM, an observation of an enhanced two-photon signal strength would indicate that all the scalar particles have masses above $63 \mathrm{GeV}$. If a substantial enhancement is observed, also upper bounds on the DM candidate and the charged scalar arise. For example, if $R_{\gamma \gamma}>1.2$, then $63 \mathrm{GeV}<M_{H} \lesssim 154 \mathrm{GeV}, 70 \mathrm{GeV}<M_{H^{ \pm}} \lesssim$ $154 \mathrm{GeV}$, and also the couplings $h H H$ and $h A A$ are strongly constrained. Combination of these results with the constraints imposed by the WMAP measurements sets even stronger bounds on the parameter space of the IDM, see [18].

\section{Acknowledgments}

We would like to thank F. Kapusta for organizing the conference and his kind support. This work was supported in part by the grant NCN OPUS 2012/05/B/ST2/03306 (2012-2016).

\section{References}

[1] ATLAS Collaboration, Measurements of Higgs boson production and couplings in diboson final states with the ATLAS detector at the LHC, Phys. Lett. B726 88 (2013) [1307.1427 [hep-ex ] ].

[2] CMS Collaboration, CMS Physics Analysis Summary, CMS PAS HIG-13-005 (2013).

[3] B. Świeżewska, M. Krawczyk, Diphoton rate in the Inert Doublet Model with a 125 GeV Higgs boson, Phys. Rev. D88, 035019 (2013) [1212.4100 [hep-ph] ].

[4] A. Arhrib, R. Benbrick, N. Gaur, $h \rightarrow \gamma \gamma$ In Inert Higgs Doublet Model, Phys. Rev. D85, 095021 (2012) [1201.2644 [hep-ph]].

[5] A. Goudelis, B. Herrmann, O. Stål, Dark matter in the Inert Doublet Model after the discovery of a Higgs-like boson at the LHC, JHEP 09, 106 (2013) [1303.3010 [hep-ph] ]. 
[6] N. G. Deshpande, E. Ma, Pattern of symmetry breaking with two Higgs doublets, Phys. Rev. D18, 2574 (1978).

[7] R. Barbieri, L. J. Hall, V. S. Rychkov, Improved Naturalness with a Heavy Higgs: An Alternative Road to LHC Physics, Phys. Rev. D74, 015007 (2006) [hep-ph/ 0603188 ].

[8] Q.-H. Cao, E. Ma, G. Rajasekaran, Observing the Dark Scalar Doublet and its Impact on the Standard-Model Higgs Boson at Colliders, Phys. Rev. D76, 095011 (2007) [0708 . 2939 [hep-ph]].

[9] I. Ginzburg, K. Kanishev, M. Krawczyk, S. Sokołowska, Evolution of Universe to the present inert phase, Phys. Rev. D82, 123533 (2010) [1009.4593 [hep-ph]].

[10] E. M. Dolle, S. Su, The Inert Dark Matter, Phys. Rev. D80, 055012 (2009) [0 906.1609 [hep-ph] ].

[11] L. Lopez Honorez, E. Nezri, J. F. Oliver, M. G. Tytgat, The Inert Doublet Model: an Archetype for Dark Matter, JCAP 0702028 (2007) [hep-ph/ 0612275 [hep-ph ] ].

[12] D. Sokołowska, (2011), Dark Matter data and constraints on quartic couplings in IDM, 1107.1991 [hep-ph].

[13] B. Świeżewska, Yukawa independent constraints for Two Higgs Doublet Models with a $125 \mathrm{GeV}$ Higgs boson, Phys. Rev. D88, 055027 (2013) [1209.5725 [hep-ph] ].

[14] K. Nakamura et al. (Particle Data Group), Review of Particle Physics, J. Phys. G37, 075021 (2010).

[15] E. Lundstrom, M. Gustafsson, J. Edsjo, Inert Doublet Model and LEP II Limits, Phys.Rev. D79, 035013 (2009) [0810.3924 [hep-ph]].

[16] M. Gustafsson, The Inert Doublet Model and its Phenomenology, in proceedings of Prospects for Charged Higgs Conference, PoS(CHARGED2010) 030 (2010) [1106.1719 [hep-ph] ].

[17] A. Djouadi, The Anatomy of Electro-Weak Symmetry Breaking. II: The Higgs bosons in the Minimal Supersymmetric Model, Phys.Rept. 459, 1 (2008) [hep-ph/0503173];

[18] M. Krawczyk, D. Sokołowska, P. Swaczyna, B. Świeżewska, Constraining Inert Dark Matter by $R_{\gamma \gamma}$ and WMAP data, JHEP 09, 055 (2013) [1305.6266 [hep-ph ] ]. 